\title{
Selective Frequency Invariant Uniform Circular Broadband Beamformer
}

\author{
Xin Zhang, ${ }^{1}$ Wee Ser, ${ }^{1}$ Zhang Zhang, ${ }^{1}$ and Anoop Kumar Krishna ${ }^{2}$ \\ ${ }^{1}$ Center for Signal Processing, Nanyang Technological University, 50 Nanyang Avenue, Singapore 639798 \\ ${ }^{2}$ EADS Innovation Works, EADS Singapore Pte Ltd., No. 41, Science Park Road, 01-30, Singapore 117610
}

Correspondence should be addressed to Xin Zhang, zhang_xin@pmail.ntu.edu.sg

Received 16 April 2009; Revised 24 August 2009; Accepted 3 December 2009

Academic Editor: Thushara Abhayapala

Copyright (C) 2010 Xin Zhang et al. This is an open access article distributed under the Creative Commons Attribution License, which permits unrestricted use, distribution, and reproduction in any medium, provided the original work is properly cited.

\begin{abstract}
Frequency-Invariant (FI) beamforming is a well known array signal processing technique used in many applications. In this paper, an algorithm that attempts to optimize the frequency invariant beampattern solely for the mainlobe, and relax the FI requirement on the sidelobe is proposed. This sacrifice on performance in the undesired region is traded off for better performance in the desired region as well as reduced number of microphones employed. The objective function is designed to minimize the overall spatial response of the beamformer with a constraint on the gain being smaller than a pre-defined threshold value across a specific frequency range and at a specific angle. This problem is formulated as a convex optimization problem and the solution is obtained by using the Second Order Cone Programming (SOCP) technique. An analysis of the computational complexity of the proposed algorithm is presented as well as its performance. The performance is evaluated via computer simulation for different number of sensors and different threshold values. Simulation results show that, the proposed algorithm is able to achieve a smaller mean square error of the spatial response gain for the specific FI region compared to existing algorithms.
\end{abstract}

\section{Introduction}

Broadband beamforming techniques using an array of microphones have been applied widely in hearing aids, teleconferencing, and voice-activated human-computer interface applications. Several broadband beamformer designs have been reported in the literature [1-3]. One design approach is to decompose the broadband signal into several narrowband signals and apply narrowband beamforming techniques for each narrowband signal [4]. This approach requires several narrowband processing to be conducted simultaneously and is computationally expensive. Another design approach is to use adaptive broadband beamformers. Such techniques use a bank of linear transversal filters to generate the desired beampattern. The filter coefficients can be derived adaptively from the received signals. One classic design example is the Frost Beamformer [5]. However, in order to have a similar beampattern over the entire frequency range, a large number of sensors and filter taps will be needed. This again leads to high computational complexity. The third approach of designing broadband beamformers is to use the Frequency-Invariant (FI) beampattern synthesis technique. As the name implies, such beamformers are designed to have constant spatial gain response over the desired frequency bands.

Over recent years, FI beamforming techniques are developed in a fast pace. It is difficult to make a distinct classification. However, in order to grasp the literature on FI beamforming in a glimpse, we classify them loosely into the following three types.

One type of FI beamformers includes those that focus on the design based on array geometry. These include, for example, the 3D sensor array design reported in [6], the rectangular sensor array design reported in [7], and the design of using subarrays in [8]. In [9], the FI beampattern is achieved by exploiting the relationship among the frequency responses of the various filters implemented at the output of each sensor.

The second type of FI beamformers is designed on the base of a least-square approach. For this type of FI beamformers, the weights of the beamformer are optimized such that the error between the actual beampattern and 
the desired beampattern is minimized over a range of frequencies. Some of such beamformers are designed in the time-frequency domain [10-12], while others are designed in the eigen-space domain [13].

The third type of FI beamformers is designed based on "Signal Transformation." For this type of beamformers, the signal received at the sensor array is transformed into a domain such that the frequency response and the spatial response of the signal can be decoupled and hence adjusted independently. This is the principle adopted in [14], where a uniform concentric circular array (UCCA) is designed to achieve the FI beampattern. Excellent results have been produced by this algorithm. One limitation of the UCCA beamformer is that a relatively large number of sensors have to be used to form the concentric circular array.

Inspired by the UCCA beamformer design, a new algorithm has been proposed by the authors of this paper and presented in [15]. The proposed algorithm attempts to optimize the FI beampattern solely for the main lobe where the signal of interest is from and relaxes the FI requirement on the side lobe. As a result, the sacrifice on performance in the undesired region is traded off for better performance in the desired region and fewer number of microphones are employed. To achieve this goal, an objective function with a quadratic constraint is designed. This constraint function allows the FI characteristic to be accurately controlled over the specified bandwidth at the expense of other parts of the spectrum which are not of concern to the designer. This objective function is formulated into a convex optimization problem and solved by SOCP readily. Our algorithm has a frequency band of interest from $0.3 \pi$ to $0.95 \pi$. If the sampling frequency is $16000 \mathrm{~Hz}$, the frequency band of interest ranges from $2400 \mathrm{~Hz}$ to $7600 \mathrm{~Hz}$. This algorithm can be applied in speech processing as the labial and fricative sounds of speech mostly lie in the 8th to 9 th octave. If the sampling frequency is $8000 \mathrm{~Hz}$, the frequency band of interest is from $1200 \mathrm{~Hz}$ to $3800 \mathrm{~Hz}$. This frequency range is useful for respiratory sounds [16].

The aim of this paper is to provide the full details of the design proposed in [15]. In addition, a computational complexity analysis of the proposed algorithm and the sensitivity performance evaluations at different numbers of sensors and different constraint parameter values are also included.

The remaining paper is organized in the following way: in Section 2, problem formulation is discussed; in Section 3, the proposed beamforming design is described; in Section 4, the design of the beamforming weight using SOCP is shown; numerical results are given in Section 5, and finally, conclusions are drawn in Section 6.

\section{Problem Formulation}

A uniformly distributed circular sensor array with $K$ number of microphones is arranged as shown in Figure 1. Each omnidirectional sensor is located at $\left(r \cos \phi_{k}, r \sin \phi_{k}\right)$, where $r$ is the radius of the circle, $\phi_{k}=2 k \pi / K$ and $k=0, \ldots, K-1$.
In this configuration, the intersensor spacing is fixed at $\lambda / 2$, where $\lambda$ is the wavelength of the signals of interest and its minimum value is denoted by $\lambda_{\min }$. The radius corresponding to $\lambda_{\min }$ is given by [14]

$$
r=\frac{\lambda_{\min }}{4 \sin (\pi / K)} .
$$

Assuming that the circular array is on a horizontal plane, the steering vector is

$$
\mathbf{a}(f, \phi)=\left[e^{j 2 \pi f r \cos \left(\phi-\phi_{0}\right) / c}, \ldots, e^{j 2 \pi f r \cos \left(\phi-\phi_{K-1}\right) / c}\right]^{T},
$$

where $T$ denotes transpose. For convenience, let $\omega$ be the normalized angular frequency, that is, $\omega=2 \pi f / f_{s}$, let $\epsilon$ be the ratio of the sampling frequency and the maximum frequency, that is, $\epsilon=f_{s} / f_{\max }$, and let $\tilde{r}$ be the normalized radius, that is, $\tilde{r}=r / \lambda_{\min }$, the steering vector can be rewritten as

$$
\mathbf{a}(\omega, \phi)=\left[e^{j \omega \tilde{r} \epsilon \cos \left(\phi-\phi_{0}\right)}, \ldots, e^{j \omega \tilde{r} \epsilon \cos \left(\phi-\phi_{K-1}\right)}\right]^{T} .
$$

Figure 2 shows the system structure of the proposed uniform circular array beamformer. The sampled signals after the sensor are represented by the vector $X[n]=$ $\left[x_{0}(n), x_{1}(n), \ldots, x_{K-1}(n)\right]^{T}$ where $n$ is the sampling instance. These sampled signals are transformed into a set of coefficients via the Inverse Discrete Fourier Transform (IDFT), where each of the coefficients is called a phase mode [17]. The $m$ th phase mode at time instance $n$ can be expressed as

$$
p_{m}[n]=\sum_{k=0}^{K-1} x_{k}[n] e^{j 2 \pi k m / K}
$$

These phase modes are passed through an FIR (Finite Impulse Response) filter where the filter coefficients are denoted as $b_{m}[n]$. The purpose of this filter is to remove the frequency dependency of the received signal $X[n]$. The beamformer output $y[n]$ is then determined as the weighted sum of the filtered signals:

$$
y[n]=\sum_{m=-L}^{L}\left(p_{m}[n] * b_{m}[n]\right) \cdot h_{m}
$$

where $h_{m}$ is the phase spatial weighting coefficients or the beamforming weights, and $*$ is the discrete-time convolution operator.

Let $M$ be the total number of phase modes and it is assumed to be an odd number. It can be seen from Figure 2 that the $K$ received signals are transformed into $M$ phase modes, where $L=(M-1) / 2$.

The corresponding spectrum of the phase modes can be obtained by taking the Discrete Time Fourier Transform 
(DTFT) of the phase modes defined in (4):

$$
\begin{aligned}
P_{m}(\omega) & =\sum_{k=0}^{K-1} X_{k}(\omega) e^{j 2 \pi k m / K} \\
& =S(\omega) \cdot \sum_{k=0}^{K-1} e^{j \omega \tilde{r} \cos \left(\phi-\phi_{k}\right)} e^{j 2 \pi k m / K},
\end{aligned}
$$

where $S(\omega)$ is the spectrum of the source signal.

Taking DTFT on both side of (5) and using (6), we have

$$
\begin{aligned}
Y(\omega) & =\sum_{m=-L}^{L} h_{m} P_{m}(\omega) B_{m}(\omega) \\
& =S(\omega) \cdot \sum_{m=-L}^{L} h_{m}\left(\sum_{k=0}^{K-1} e^{j \omega \tilde{r} \cos \left(\phi-\phi_{k}\right)} e^{j 2 \pi k m / K}\right) B_{m}(\omega) .
\end{aligned}
$$

Consequently, the response of the beamformer can be expressed as

$$
G(\omega, \phi)=\sum_{m=-L}^{L} h_{m}\left(\sum_{k=0}^{K-1} e^{j \omega \tilde{r} \cos \left(\phi-\phi_{k}\right)} e^{j 2 \pi k m / K}\right) B_{m}(\omega) .
$$

In order to obtain an FI response, terms which are functions of $\omega$ are grouped together using the Jacobi-Anger expansion given as follows [18]:

$$
e^{j \beta \cos \gamma}=\sum_{n=-\infty}^{+\infty} j^{n} J_{n}(\beta) e^{j n \gamma},
$$

where $J_{n}(\beta)$ is the Bessel function of the first kind of order $n$.

Substituting (9) into (8), and applying property of the Bessel function, the spatial response of the beamformer can now be approximated by

$$
G(\omega, \phi)=\sum_{m=-L}^{L} h_{m} \cdot e^{j m \phi} \cdot K \cdot j^{m} \cdot J_{m}(\omega \widetilde{r}) \cdot B_{m}(\omega) .
$$

This process has been described in [13] and its detailed derivation can be found in [14].

\section{Proposed Novel Beamformer}

With the above formulation, we propose the following beam pattern synthesis method. The basic idea is to enhance the broadband signals for a specific frequency region and at a certain direction. In order to achieve this goal, the following



Figure 1: Uniform Circular Array Configuration.

objective function is proposed:

$$
\begin{array}{ll}
\min & \iint_{\omega}\|G(\omega, \phi)\|^{2} d \omega d \phi, \\
\text { s.t. } & \left\|G\left(\omega, \phi_{0}\right)-1\right\| \leq \delta, \quad \omega \in\left[\omega_{l}, \omega_{u}\right],
\end{array}
$$

where $G(\omega, \phi)$ is the spatial response of the beamformer given in (10), and $\omega_{l}$ and $\omega_{u}$ are the lower and upper limit of the specified frequency region respectively. $\phi_{0}$ is the specified direction and $\delta$ is a predefined threshold value that controls the magnitude of the ripples of the main beam.

In principle, the objective function defined above aims to minimize the square of the spatial gain response across all frequencies and all angles, while constraining the gain to the value of one at the specified angle. This is to relax the gain constraint to one angle instead of all angles, so that the FI beampattern in the specified region can be improved. With this constraint setting, the resulting beamformer can enhance broadband desired signals arriving from one direction while attenuate broadband noise received from other directions. The concept for formulating the objective function is similar to Capon beamformer [19]. One difference is that the Capon beamformer aims to minimize the data dependent array output power at a single frequency, while the proposed algorithm aims to minimize the data independent array output power across a wide range of frequencies. Another difference is that the constraint used in Capon beamformer is a hard constraint, whereas the array gain used in the proposed algorithm is a soft constraint, which can result in a higher degree of flexibility.

The proposed algorithm is expected to have lower computational complexity compared to the UCCA beamformer. The later is designed to achieve FI beampattern for all angles whereas the proposed algorithm focuses only on a specified angle. For the same reason, the proposed algorithm is expected to have a larger degree of freedom too. This explains the result in having a better FI beampattern for a given number of sensors. These performance improvements have been supported by computer simulations and will be discussed in the later part of this paper. 




FIgURE 2: The system structure of a uniform circular array beamformer.

The optimization problems defined by (10) and (11) require the optimum values of both the compensation filter and the spatial weightings to be determined simultaneously. As such, Cholesky factorization is used to transform the objective function further into the Second-Order Cone Programming (SOCP) problem. The details of implementation will be discussed in the following section. It should be noted that when the threshold value $\delta$ equals zero, the optimization process becomes a linearly constrained problem.

\section{Convex Optimization-Based Implementation}

Second-Order Cone Programming (SOCP) is a popular tool for solving convex optimization problem, and it has been used for array pattern synthesis problem [20-22] since the early papers by Lobo et al. [23]. One advantage of SOCP is that the global optimal solution is guaranteed if it exists, whereas constrained least square optimization procedure looks for local minimum. Another important advantage is that it is very convenient to include additional linear or convex quadratic constraints, such as the norm constraint of the variable vector, in the problem formulation. The standard form of SOCP can be written as follows:

$$
\begin{array}{ll}
\min & \mathbf{b}^{T} \mathbf{x}, \\
\text { s.t. } & \mathbf{d}_{i}^{T} \mathbf{x}+q_{i} \geq\left\|\mathbf{A}_{i} \mathbf{x}+\mathbf{c}_{i}\right\|_{2}, \quad i=1, \ldots, N,
\end{array}
$$

where $\mathbf{x} \in R^{m}$ is the variable vector; the parameters are $\mathbf{b} \in$ $R^{m}, \mathbf{A}_{i} \in R^{\left(n_{i}-1\right) \times m}, \mathbf{c}_{i} \in R^{n_{i}-1}, \mathbf{d}_{i} \in R^{m}$, and $q_{i} \in R$. The norm appearing in the constraints is the standard Euclidean norm, that is, $\|u\|_{2}=\left(u^{T} u\right)^{1 / 2}$.

4.1. Convex Optimization of the Beampattern Synthesis Problem. The following transformations are carried out to convert (11) into the standard form defined by (12).

First, $B_{m}(\omega)=\sum_{n=0}^{N_{m}} b_{m}[n] e^{-j n \omega}$ is substituted into (10), where $N_{m}$ is the filter order for each phase. The spatial response of the beamformer can now be expressed as

$$
G(\omega, \phi)=\sum_{m=-L}^{L} h_{m} \cdot e^{j m \phi} \cdot K \cdot j^{m} \cdot J_{m}(\omega \tilde{r}) \cdot\left[\sum_{n=0}^{N_{m}} b_{m}[n] e^{-j n \omega}\right] .
$$

Using the identity $e^{-j n \omega}=\cos (n \omega)-j \sin (n \omega),(13)$ becomes

$$
\begin{aligned}
G(\omega, \phi)= & \sum_{m=-L}^{L} h_{m} \cdot e^{j m \phi} \cdot K \cdot j^{m} \cdot J_{m}(\omega \tilde{r}) \\
& \cdot\left[\sum_{n=0}^{N_{m}} b_{m}[n](\cos (n \omega)-j \sin (n \omega)]\right. \\
= & K \sum_{m=-L}^{L} h_{m} \cdot e^{j m \phi} \cdot j^{m} \cdot J_{m}(\omega \tilde{r}) \\
& \cdot\left[\sum_{n=0}^{N_{m}}\left(b_{m}[n] \cos (n \omega)-j b_{m}[n] \sin (n \omega)\right)\right] \\
= & K \sum_{m=-L}^{L} h_{m} \cdot e^{j m \phi} \cdot j^{m} \cdot J_{m}(\omega \tilde{r}) \\
& \left.\cdot\left[\sum_{n=0}^{N_{m}}\left(b_{m}[n] \cos (n \omega)-j \sum_{n=0}^{N_{m}} b_{m}[n] \sin (n \omega)\right)\right)\right] \\
= & K \sum_{m=-L}^{L} h_{m} \cdot e^{j m \phi} \cdot j^{m} \cdot J_{m}(\omega \tilde{r}) \cdot\left[\mathbf{c}_{m} \overline{\mathbf{b}}_{m}-j \mathbf{s}_{m} \overline{\mathbf{b}}_{m}\right],
\end{aligned}
$$

where $\overline{\mathbf{b}}_{m}=\left[b_{m}[0], b_{m}[1], \ldots, b_{m}\left[N_{m}\right]\right]^{T} ; \mathbf{c}_{m}=[\cos (0)$, $\left.\cos (\omega), \ldots, \cos \left(N_{m} \cdot \omega\right)\right] ; \mathbf{s}_{m}=\left[\sin (0), \sin (\omega), \ldots, \sin \left(N_{m}\right.\right.$. $\omega)]$.

$h_{m}$ is the spatial weighting in the system structure, and $\overline{\mathbf{b}}_{m}$ is the FIR filter coefficient vector for each phase.

Let $\mathbf{u}_{m}=h_{m} \cdot j^{m} \cdot \overline{\mathbf{b}}_{m}$, we have

$$
\begin{aligned}
G(\omega, \phi)= & K \sum_{m=-L}^{L} e^{j m \phi} \cdot J_{m}(\omega \widetilde{r}) \cdot \mathbf{c}_{m} \mathbf{u}_{m} \\
& -j \cdot K \sum_{m=-L}^{L} e^{j m \phi} \cdot J_{m}(\omega \tilde{r}) \cdot \mathbf{s}_{m} \mathbf{u}_{m} \\
= & \overline{\mathbf{c}}(\omega, \phi) \overline{\mathbf{u}}-j \overline{\mathbf{s}}(\omega, \phi) \overline{\mathbf{u}},
\end{aligned}
$$

where $\overline{\mathbf{c}}(\omega, \phi)=\left[K e^{j(-L) \phi} J_{-L}(\omega \widetilde{r}) \mathbf{c}_{-L}, \ldots, K e^{j(L) \phi} J_{L}(\omega \widetilde{r}) \mathbf{c}_{L}\right]$; $\overline{\mathbf{u}}=\left[\mathbf{u}_{-L}^{T}, \mathbf{u}_{-L+1}^{T}, \ldots, \mathbf{u}_{L}^{T}\right]^{T} ; \overline{\mathbf{s}}(\omega, \phi)=\left[K e^{j(-L) \phi} J_{-L}(\omega \tilde{r}) \mathbf{s}_{-L}\right.$, $\left.\ldots, K e^{j(L) \phi} J_{L}(\omega \widetilde{r}) \mathbf{s}_{L}\right]$. 
Representing the complex spatial response $G(\omega, \phi)$ by a 2-dimensional vector $\mathbf{g}(\omega, \phi)$ which display the real and imaginary parts into rows of a vector separately, (15) is rewritten in the following form:

$$
\mathbf{g}(\omega, \phi)=\left(\begin{array}{c}
\overline{\mathbf{c}}(\omega, \phi) \\
-\overline{\mathbf{s}}(\omega, \phi)
\end{array}\right) \overline{\mathbf{u}}=\mathbf{A}(\omega, \phi)^{H} \overline{\mathbf{u}}
$$

Hence, $\|G(\omega, \phi)\|^{2}=\mathbf{g}^{H} \mathbf{g}=\left(\mathbf{A}(\omega, \phi)^{H} \overline{\mathbf{u}}\right)^{H}\left(\mathbf{A}(\omega, \phi)^{H} \overline{\mathbf{u}}\right)$ $=\overline{\mathbf{u}}^{H} \mathbf{A}(\omega, \phi) \mathbf{A}(\omega, \phi)^{H} \overline{\mathbf{u}}$.

The objective function and the constraint inequality defined in (11) can now be written as

$$
\begin{array}{ll}
\min _{\overline{\mathbf{u}}} & \overline{\mathbf{u}}^{H} \mathbf{R} \overline{\mathbf{u}}, \\
\text { s.t. } & \left\|G\left(\omega, \phi_{0}\right)-1\right\| \leq \delta, \quad \text { for } \omega \in\left[\omega_{l}, \omega_{u}\right],
\end{array}
$$

where $\mathbf{R}=\int_{\omega} \int_{\phi} \mathbf{A}(\omega, \phi) \mathbf{A}(\omega, \phi)^{H} d \omega d \phi$.

In order to transform (17) into the SOCP form defined by (12), the cost function must be a linear equation. Since matrix $R$ is hermitian and positive definite, it can be decomposed into an upper triangular matrix and its transpose using Cholesky factorization, that is, $\mathbf{R}=\mathbf{D}^{H} \mathbf{D}$, where $\mathbf{D}$ is the Cholesky factorization of $\mathbf{R}$. Substituting this into (17), we have

$$
\overline{\mathbf{u}}^{H} \mathbf{R} \overline{\mathbf{u}}=\overline{\mathbf{u}}^{H}\left(\mathbf{D}^{H} \mathbf{D}\right) \overline{\mathbf{u}}=(\mathbf{D} \overline{\mathbf{u}})^{H}(\mathbf{D} \overline{\mathbf{u}}) .
$$

This further simplifies (17) into the following form:

$$
\begin{array}{ll}
\min _{\overline{\mathbf{u}}} & d^{2}, \\
\text { s.t. } & \left\{\begin{array}{l}
d^{2}=\|\mathbf{D} \cdot \overline{\mathbf{u}}\|^{2}, \\
\left\|G\left(\omega, \phi_{0}\right)-1\right\| \leq \delta \quad \text { for } \omega \in\left[\omega_{l}, \omega_{u}\right] .
\end{array}\right.
\end{array}
$$

Denoting $t$ as the maximum norm of vector $\|\mathbf{D} \overline{\mathbf{u}}\|$ subject to various choices of $\overline{\mathbf{u}}$, (19) reduces to

$$
\begin{array}{ll}
\min _{\overline{\mathbf{u}}} & t, \\
\text { s.t. } & \left\{\begin{array}{l}
\|\mathbf{D} \cdot \overline{\mathbf{u}}\| \leq t, \\
\left\|G\left(\omega, \phi_{0}\right)-1\right\| \leq \delta \quad \text { for } \omega \in\left[\omega_{l}, \omega_{u}\right] .
\end{array}\right.
\end{array}
$$

It should be noted that (20) contains I different constraints where $I$ uniformly divides the frequency range spanned by $\omega$.

Lastly, in order to solve (20) by SOCP toolbox, we stack $t$ and the coefficients of $\overline{\mathbf{u}}$ together and define $\mathbf{y}=[t ; \overline{\mathbf{u}}]$. Let $\mathbf{a}=[1, \mathbf{0}]^{T}$, so that $t=\mathbf{a}^{T} \mathbf{y}$. As a result, the objective function and the constraint defined in (11) can be expressed as

$$
\begin{array}{ll}
\min _{\overline{\mathbf{y}}} & \mathbf{a}^{T} \mathbf{y}, \\
\text { s.t. } & \begin{cases}\left\|\left[\begin{array}{ll}
\mathbf{0} & \mathbf{D}
\end{array}\right] \mathbf{y}\right\| \leq \mathbf{a}^{T} \mathbf{y}, \\
\left\|\left[\begin{array}{ll}
\mathbf{0} & \mathbf{A}\left(\omega, \phi_{0}\right)^{H}
\end{array}\right] \mathbf{y}-\left(\begin{array}{l}
1 \\
0
\end{array}\right)\right\| \leq \delta \quad \text { for } \omega \in\left[\begin{array}{ll}
\omega_{l} & \omega_{u}
\end{array}\right],\end{cases}
\end{array}
$$

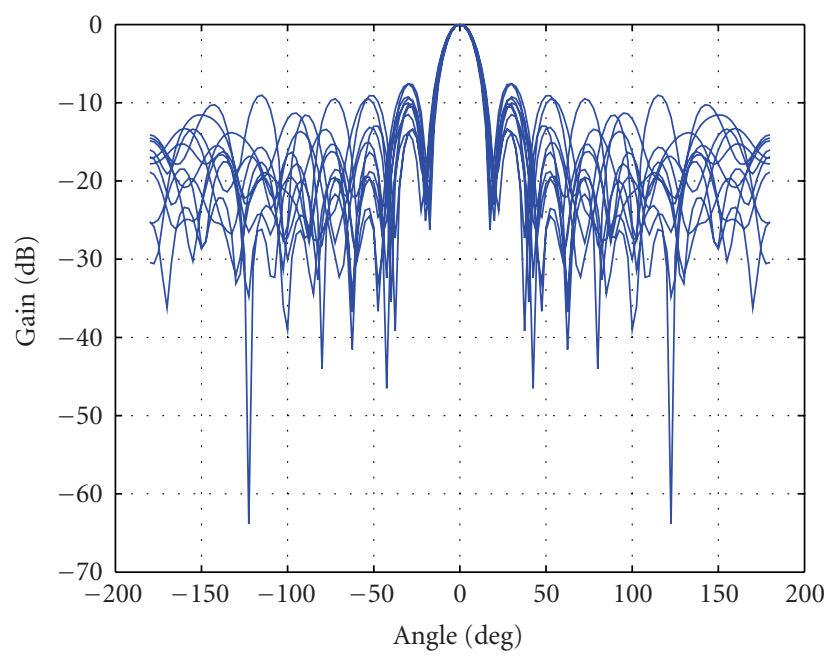

Figure 3: The normalized spatial response of the proposed beamformer for $\omega=[0.3 \pi, 0.95 \pi]$.

where $\mathbf{0}$ is the zero matrix with its dimension determined from the context.

Equation (21) can now be solved using convex optimization toolbox such as SeDuMi [24] with great efficiency.

4.2. Computational Complexity. When the Interior-Point Method (IPM) is used to solve the SOCP problem defined in (21), the number of iterations needed is bounded by $O(\sqrt{N})$ where $N$ is the number of constraints. The amount of computation per iteration is $O\left(n^{2} \sum_{i} n_{i}\right)$ [23].

The bulk of the computational requirement of the broadband array pattern synthesis comes from the optimization process. The computational complexity of the optimization process of the proposed algorithm and that of the UCCA algorithm have been calculated and are listed in Table 1.

It can be seen from Table 1 that the proposed algorithm requires a similar amount of computation per iterations but a much smaller number of iterations compared to the UCCA algorithm. The overall computational load of the proposed method is therefore much smaller that that is needed by the UCCA algorithm. It should be noted that, as the coefficients are optimized in the phase modes, the comparative computational load presented above is calculated based on the number of phase modes and not the number of sensors. Nevertheless, the larger the number of sensors, the larger the number of phase modes too.

\section{Numerical Results}

In this numerical study, the performance of the proposed beamformer is compared with that of UCCA beamformer [14] and Yan's beamformer [25], for the specified frequency region. The evaluation metric used to quantify the frequency invariance (FI) characteristics is the mean squared error of the array gain variation at the specified direction. The sensitivity performance of the proposed algorithm will also be evaluated for different number of sensors and different 
TABLE 1: Computational complexity of different broadband beampattern synthesis method.

\begin{tabular}{lcc}
\hline Method & Number of iteration & Amount of computation per iteration \\
\hline UCCA & $O\{\sqrt{I \times M}\}$ & $O\left\{(1+P(1+N m))^{2}[2 M(I+1)]\right\}$ \\
Equation (11) & $O\{\sqrt{1+I}\}$ & $O\left\{\left[M(N m+1)^{2}\right][2 I+M(N m+1)+1]\right\}$ \\
\hline
\end{tabular}

TABle 2: Comparison of array gain at each frequency along the desired direction for the three methods.

\begin{tabular}{llll}
\hline $\begin{array}{l}\text { Normalized } \\
\begin{array}{l}\text { Frequency } \\
\text { (radians/sample) }\end{array}\end{array}$ & $\begin{array}{l}\text { Proposed } \\
\text { Beamformer } \\
(\mathrm{dB})\end{array}$ & $\begin{array}{l}\text { Yan's } \\
\text { beamformer } \\
(\mathrm{dB})\end{array}$ & $\begin{array}{l}\text { UCCA } \\
\text { Beamformer } \\
(\mathrm{dB})\end{array}$ \\
\hline 0.3 & -0.0007 & 0 & 0.6761 \\
0.4 & -0.0248 & -0.8230 & 0.1760 \\
0.5 & 0.0044 & -1.3292 & -0.022 \\
0.6 & -0.0097 & -1.6253 & -0.2186 \\
0.7 & -0.0046 & -1.8789 & -0.6301 \\
0.8 & 0.0085 & -2.9498 & -0.1291 \\
0.9 & -0.0033 & -6.2886 & 0.1477 \\
\hline
\end{tabular}

threshold values set for magnitude control of the ripples of the main beam.

A uniform circular array consisting of 20 sensors is considered. All the sensors are assumed perfectly calibrated. The number of phase modes $\mathrm{M}$ is set to be 17 and thus there are 17 spatial weighting coefficients. The order of the compensation filter is set to be 16 for all the phase modes. The frequency region of interest is specified to be from $0.3 \pi$ to $0.95 \pi$. The threshold value, $\delta$, which controls the magnitude of the ripples of the main beam is set to 0.1 . The specified direction is set to be $0^{\circ}$ where the reference microphone is located.

There are several optimization criteria presented in [25]. The one that is chosen to compare is peak sidelobe constrained minimax mainlobe spatial response variation (MSRV) design. Its objective is to minimize the maximum MSRV with peak sidelobe constraint. The mathematic expression is shown as follows:

$$
\begin{array}{ll}
\min _{\mathbf{h}} & \sigma, \\
\text { s.t. } & \left\{\begin{array}{l}
\mathbf{u}^{T}\left(f_{0}, \phi_{0}\right) \mathbf{h}=1, \\
\left|\left[\mathbf{u}\left(f_{k}, \theta_{q}\right)-\mathbf{u}\left(f_{0}, \theta_{q}\right)\right]^{T} \mathbf{h}\right| \leq \sigma, \\
\left|\mathbf{u}\left(f_{k}, \theta_{s}\right)^{T} \mathbf{h}\right| \leq \varepsilon, \\
f_{k} \in\left[f_{l}, f_{u}\right], \quad \theta_{q} \in \Theta_{\mathrm{ML}}, \quad \theta_{s} \in \Theta_{\mathrm{SL}},
\end{array}\right.
\end{array}
$$

where $f_{0}$ is the reference frequency and choose to have the value of $f_{l}$, and $\mathbf{h}$ is the beamformed weightings to be optimized. $\varepsilon$ is the peak sidelobe constraint and set to be 0.036. $\Theta_{\mathrm{ML}}$ and $\Theta_{\mathrm{SL}}$ represent the mainlobe and sidelobe region, respectively.

The beampattern obtained for the proposed beamformer for the frequency region of interest is shown in Figure 3. The spatial response of the proposed beamformer at 10 uniformly

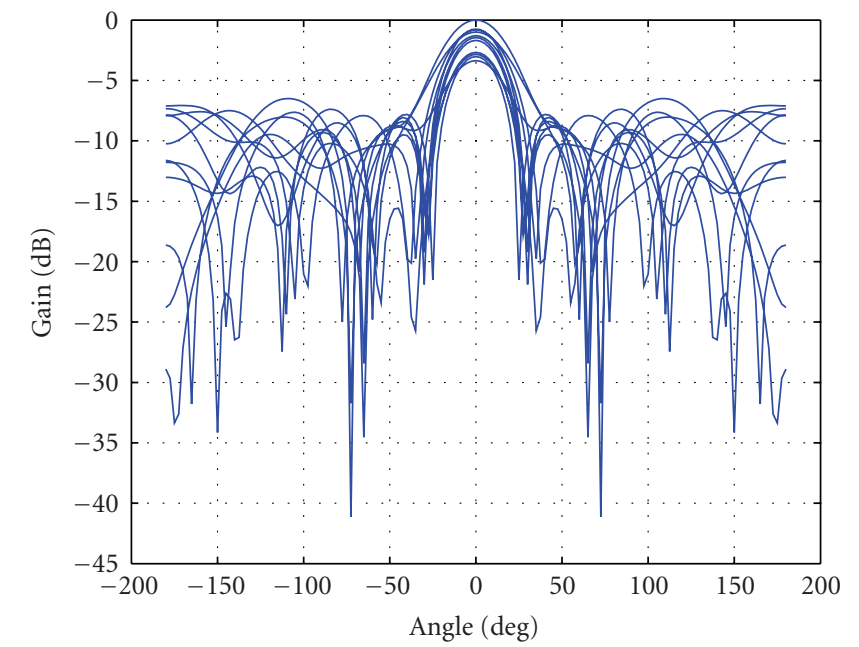

Figure 4: The normalized spatial response of the UCCA beamformer for $\omega=[0.3 \pi, 0.95 \pi]$.

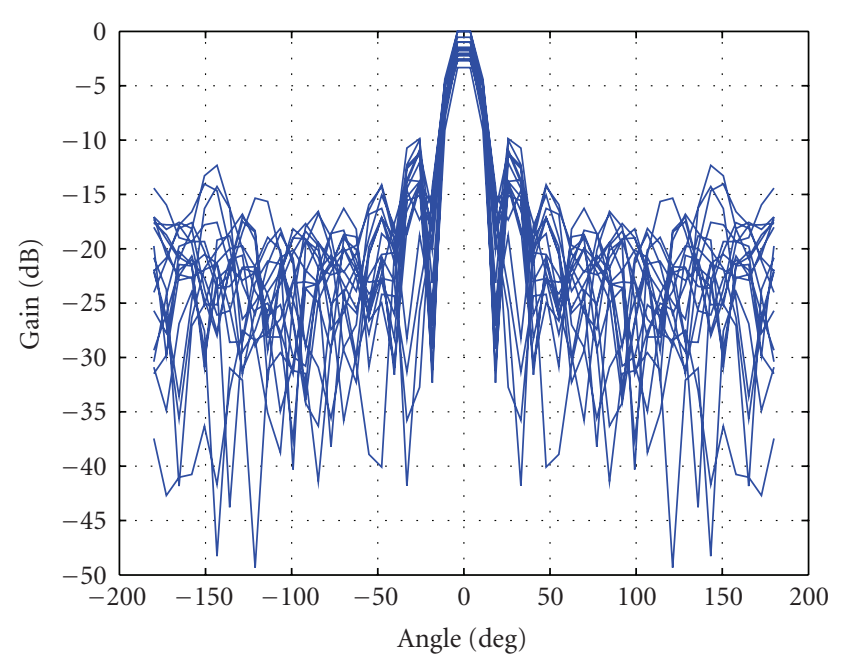

FIGURE 5: The normalized spatial response of Yan's beamformer for $\omega=[0.3 \pi, 0.95 \pi]$.

spaced discrete frequencies is superimposed. It can be seen that, the proposed beamformer has approximately a constant gain within the frequency region of interest in the specified direction $\left(0^{\circ}\right)$. As the direction deviates from $0^{\circ}$, the FI property becomes poorer. The peak sidelobe level has a value of $-8 \mathrm{~dB}$.

The beampattern of the UCCA beamformer is shown in Figure 4 . As the proposed algorithm is based on a circular array, only one layer of the UCCA concentric array is used for the numerical study. All other parameter settings remain the same as that used for the proposed algorithm. As shown 




Figure 6: Comparison on FI characteristic between the proposed beamformer, UCCA beamformer and Yan's beamformer at 0 degree for $\omega=[0.3 \pi, 0.95 \pi]$.



FIGURE 7: Directivity versus frequency for the broadband beam pattern shown in Figure 3.

in the figure, the beampattern of the UCCA beamformer is not as constant as that of the proposed beamformer in the specified direction $\left(0^{\circ}\right)$. The peak sidelobe level which has a value of $-6 \mathrm{~dB}$ is higher as compared to the proposed beamformer too.

The beampattern of Yan's beamformer is shown in Figure 5. The frequency invariant characteristics is poorer at the desired direction. However it has the lowest sidelobe level among all. From this comparison, we find that having processed the signal in phase mode, the frequency range for the beamformer to achieve Frequency Invariant (FI) characteristics is wider.

The mean squared errors of the spatial response gain in the specified direction and across different frequencies for



FIGURE 8: White noise gain versus frequency for the broadband beam pattern shown in Figure 3.

different methods are shown in Figure 6. It is seen that the proposed beamformer outperforms both the UCCA beamformer and Yan's beamformer on achieving FI characteristic at the desired direction. Table 2 tabulates the array gain at each frequency along the desired direction for these three methods.

Furthermore, the performance of the frequency invariant beam pattern obtained by the proposed method is assessed by evaluating the directivity and the white-noise gain over the entire frequency band considered, as shown in Figures 7 and 8 , respectively. Directivity describes the ability of the array to suppress a diffuse noise field, while white noise gain shows the ability of the array to suppress spatially uncorrelated noise, which can be caused by self-noise of the sensors. Because our array is a circular array, the directivity $D(\omega)$ is calculated using the following equation:

$$
D(\omega)=\frac{\left|\sum_{m=-L}^{L} B_{m}(\omega)\right|^{2}}{\sum_{m=-L}^{L} \sum_{n=-L}^{L} B_{m}(\omega) B_{n}(\omega)^{\prime} \operatorname{sinc}[(m-n) 2 \pi \omega r / c]},
$$

where $B_{m}(\omega)$ is the frequency response of the FIR filter at $m$ th phase mode, and $r$ is the radius of the circle.

As shown in the figure, the directivity has a constant profile, with an average value equal to $13.1755 \mathrm{~dB}$. The white noise gain ranges from $5.5 \mathrm{~dB}$ to $11.3 \mathrm{~dB}$. These positive values represent an attenuation of self-noise of the microphones. As expected, the lower the frequency, the smaller the white noise gain, and the higher the sensitivity to array imperfections. Hence, the proposed beamformer is more sensitive to array imperfection at low frequency and is the most robust to array imperfection at normalized frequency $0.75 \pi$.

5.1. Sensitivity Study-Number of Sensors. Most FI beamformers reported in the literature employ a large number of sensors. In this study, the number of sensors used 


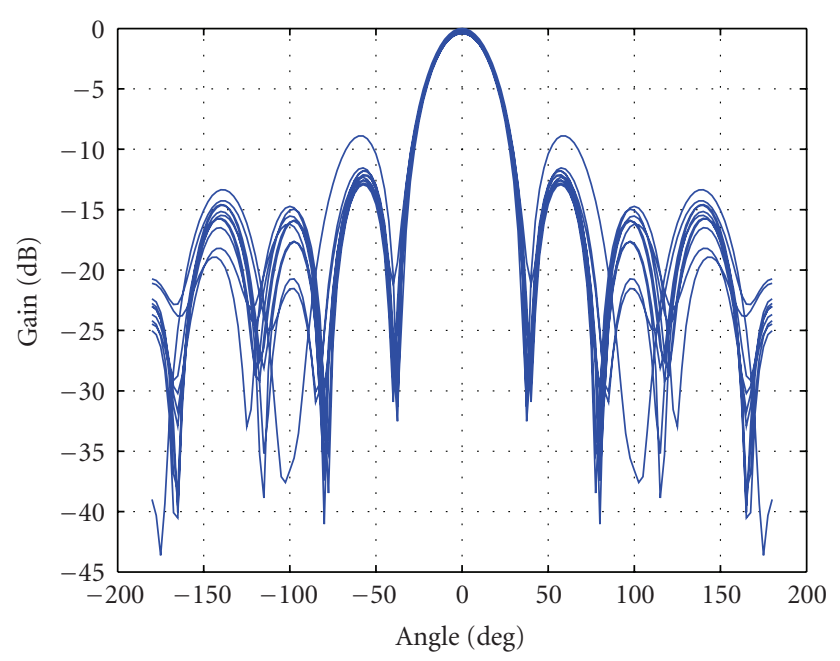

FIGURE 9: The normalized spatial response of the proposed FI beamformer for 10 microphones.

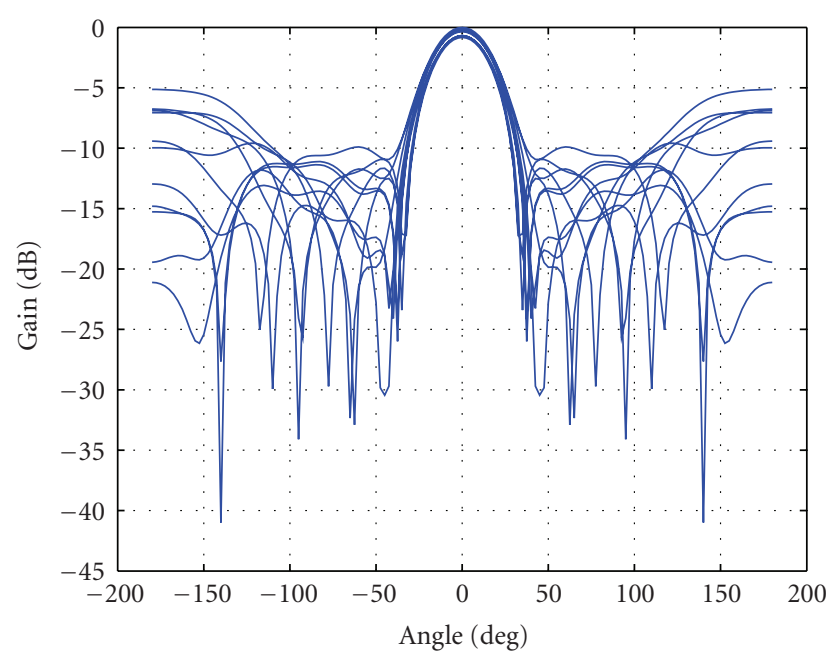

Figure 10: The normalized spatial response of the UCCA beamformer for 10 microphones.

are reduced from 20 to 10 and 8 and the performances of the proposed FI beamformer, UCCA beamformer, and Yan's beamformer are compared. The results are shown in Figures $9,10,11,12,13$, and 14 . As seen from the simulations, when 10 microphones are employed, the proposed algorithm achieves the best FI performance in the mainlobe region, with a sidelobe level of $-8 \mathrm{~dB}$. For UCCA method and Yan's method, frequency invariant characteristics are not promising at the desired direction, and higher sidelobes are obtained. When the number of microphone is further reduced to 8 , our proposed method is still able to produce reasonable FI beampattern whereas the FI property of the beampattern of the UCCA algorithm becomes much poorer in the specified direction.

5.2. Sensitivity Study-Different Threshold Value $\delta$. In this proposed algorithm, $\delta$ is a parameter created to define



FIGURE 11: The normalized spatial response of the Yan's beamformer for 10 microphones.

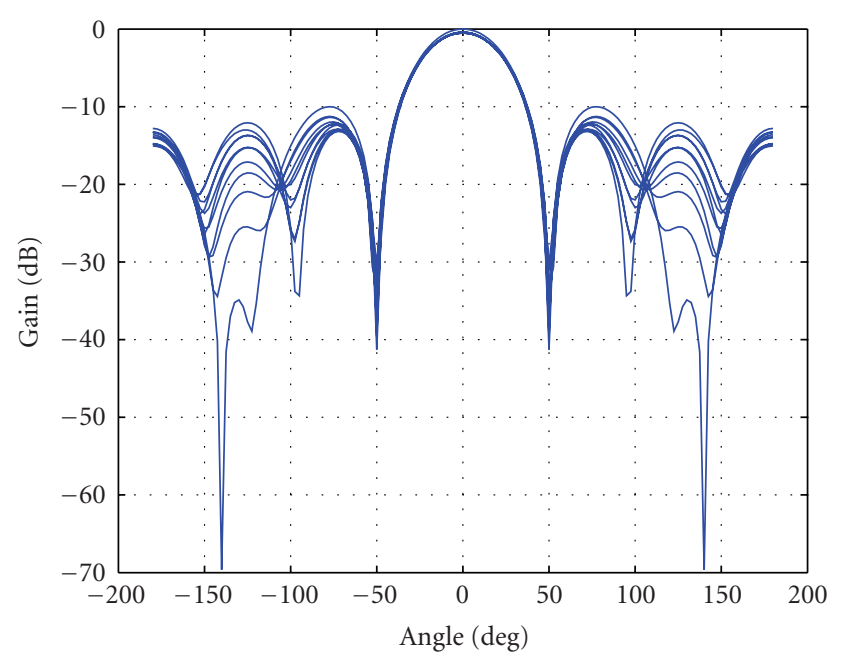

Figure 12: The normalized spatial response of the proposed FI beamformer for 8 microphones.

the allowed ripples in the magnitude of the main beam spatial gain response. In this section, different values of $\delta$ are used to study the sensitivity of the performance of the proposed algorithm to this parameter value. Three values, namely, $\delta=[0.001,0.01,0.1]$ are selected and the results obtained are shown in Figures 15, 16, and 17, respectively. The specified frequency region of interest remains the same. Figure 18 shows the mean squared error of the array gain at the specified direction $\left(0^{\circ}\right)$ for the three different $\delta$ values studied.

As shown in the figures, as the value of $\delta$ decreases, the FI performance at the specified direction improves. The results also show that the improvement in the FI performance in the specified direction is achieved with an increase in the peak sidelobe level and a poorer FI beampattern in the other directions in the main beam. For example, when the value of $\delta$ is 0.001 , the peak sidelobe of the spatial response is 


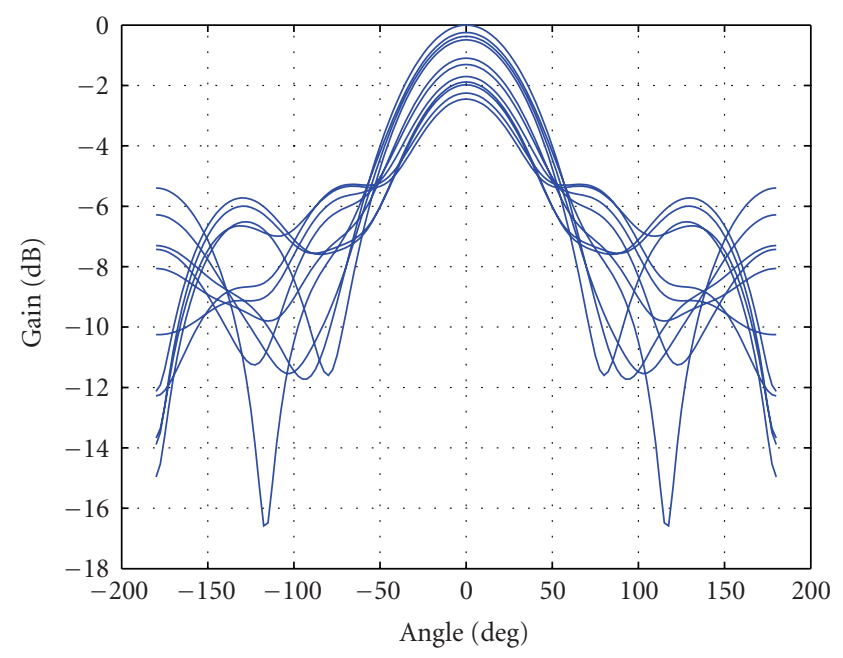

FIGURE 13: The normalized spatial response of the UCCA beamformer for 8 microphones.

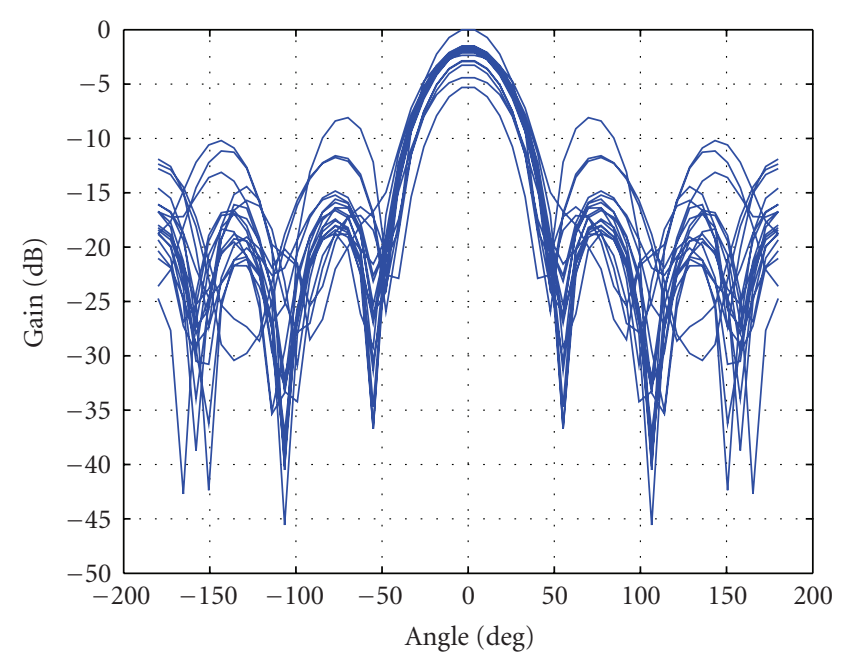

FIgURE 14: The normalized spatial response of the Yan's beamformer for 8 microphones.

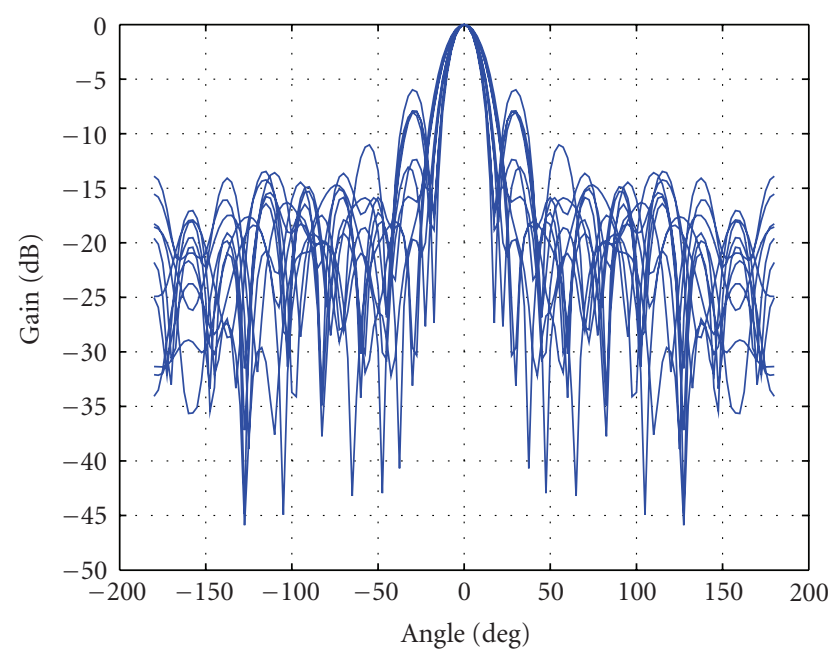

FIGURE 15: The normalized spatial response of the proposed beamformer for $\delta=0.001$.

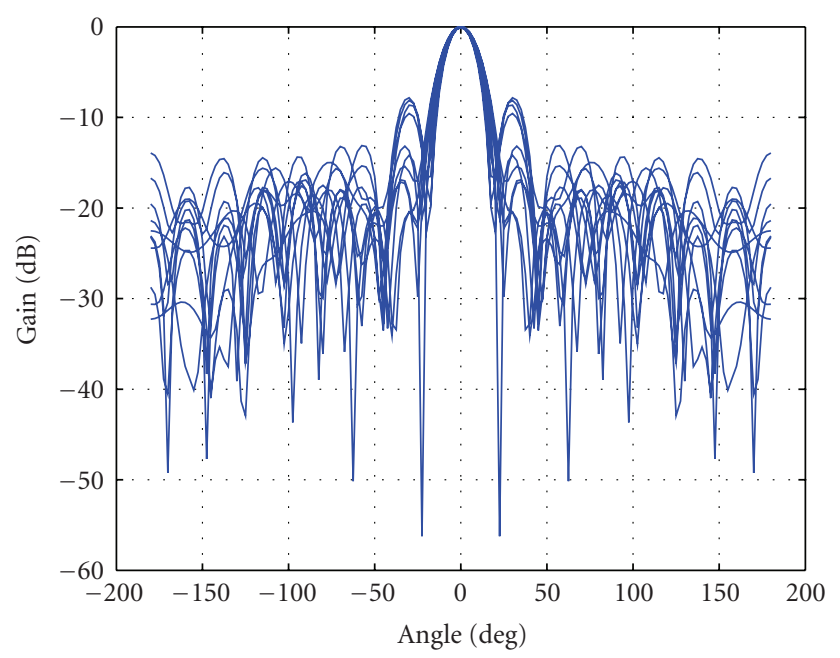

FIGURE 16: The normalized spatial response of the proposed beamformer for $\delta=0.01$.

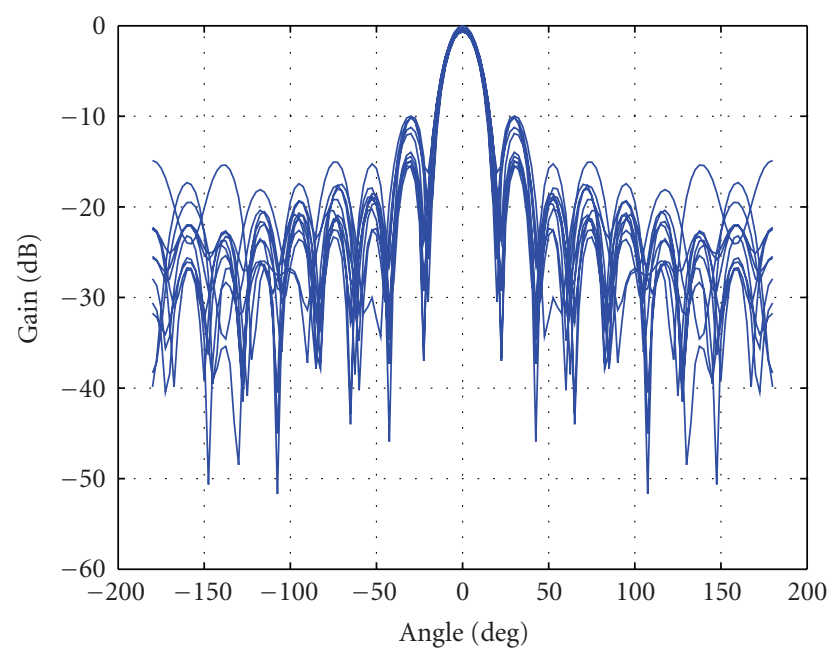

FIGURE 17: The normalized spatial response of the proposed beamformer for $\delta=0.1$.

as high as $-5 \mathrm{~dB}$ and the beampatterns do not overlap well in the main beam. As $\delta$ increases to 0.1 , the peak sidelobe of the spatial response is approximately $-10 \mathrm{~dB}$ (lower) and the beampatterns in the main beam are observed to have a relatively good FI characteristics.

\section{Conclusion}

A selective frequency invariant uniform circular broadband beamformer is presented in this paper. Other than providing the details of a recent conference paper presented by the authors of this paper, a complexity analysis and two sensitivity studies on the proposed algorithm are also presented in this paper. The proposed algorithm is designed to minimize an objective function of the spatial response gain with a constraint on the gain being smaller than a predefined threshold value across a specified frequency range and in a specified direction. The problem is formulated as a convex 




FIGURE 18: Comparison on FI characteristic of the proposed beamformer for $\delta=0.001,0.01$ and 0.1 at 0 degree for $\omega=$ $[0.3 \pi, 0.95 \pi]$.

optimization problem and the solution is obtained by using the Second-Order Cone Programming (SOCP) technique. The complexity analysis shows that the proposed algorithm has a lower computational requirement compared to that of the UCCA algorithm for the problem defined. Numerical results show that the proposed algorithm is able to achieve a more FI beampattern and a smaller mean square error of the spatial response gain in the specified direction across the specified FI region compared to the UCCA algorithm.

\section{Acknowledgments}

The authors would like to acknowledge the helpful discussions given by $\mathrm{H}$. $\mathrm{H}$. Chen of the University of Hong Kong on the UCCA algorithm. The authors would also like to thank STMicroelectronics (Singapore) for the sponsorship of this project. Last but not the least, the authors would like to thank the reviewers too for their constructive comments and suggestions which greatly improve the quality of this manuscript.

\section{References}

[1] H. Krim and M. Viberg, "Two decades of array signal processing research: the parametric approach," IEEE Signal Processing Magazine, vol. 13, no. 4, pp. 67-94, 1996.

[2] D. H. Johnson and D. E. Dudgeon, Array Signal Processing: Concepts and Techniques, Prentice-Hall, Upper Saddle River, NJ, USA, 1993.

[3] R. A. Monzingo and T. W. Miller, Introduction to Adaptive Arrays, John Wiley \& Sons, SciTech, New York, NY, USA, 2004.

[4] B. D. Van Veen and K. M. Buckley, "Beamforming: a versatile approach to spatial filtering," in Proceedings of the IEEE Transactions on Acoustics, Speech, and Signal Processing (ICASSP '88), April 1988.
[5] O. L. Frost III, "An algorithm for linearly constrained adaptive array processing," Proceedings of the IEEE, vol. 60, no. 8, pp. 926-935, 1972.

[6] W. Liu, D. McLernon, and M. Ghogho, "Frequency invariant beamforming without tapped delay-lines," in Proceedings of the IEEE International Conference on Acoustics, Speech, and Signal Processing (ICASSP '07), vol. 2, pp. 997-1000, Honolulu, Hawaii, USA, April 2007.

[7] M. Ghavami, "Wideband smart antenna theory using rectangular array structures," IEEE Transactions on Signal Processing, vol. 50, no. 9, pp. 2143-2151, 2002.

[8] T. Chou, "Frequency-independent beamformer with low response error," in Proceedings of the 20th IEEE Transactions on Acoustics, Speech, and Signal Processing (ICASSP '95), vol. 5, pp. 2995-2998, Detroit, Mich, USA, May 1995.

[9] D. B. Ward, R. A. Kennedy, and R. C. Williamson, "FIR filter design for frequency invariant beamformers," IEEE Signal Processing Letters, vol. 3, no. 3, pp. 69-71, 1996.

[10] A. Trucco and S. Repetto, "Frequency invariant beamforming in very short arrays," in Proceedings of the MTS/IEEE TechnoOcean (Oceans '04), vol. 2, pp. 635-640, November 2004.

[11] A. Trucco, M. Crocco, and S. Repetto, "A stochastic approach to the synthesis of a robust frequency-invariant filter-andsum beamformer," IEEE Transactions on Instrumentation and Measurement, vol. 55, no. 4, pp. 1407-1415, 2006.

[12] S. Doclo and M. Moonen, "Design of far-field and near-field broadband beamformers using eigenfilters," Signal Processing, vol. 83, no. 12, pp. 2641-2673, 2003.

[13] L. C. Parra, "Least squares frequency-invariant beamforming," in Proceedings of the IEEE Workshop on Applications of Signal Processing to Audio and Acoustics, pp. 102-105, New Paltz, NY, USA, October 2005.

[14] S. C. Chan and H. H. Chen, "Uniform concentric circular arrays with frequency-invariant characteristics-theory, design, adaptive beamforming and DOA estimation," IEEE Transactions on Signal Processing, vol. 55, no. 1, pp. 165-177, 2007.

[15] X. Zhang, W. Ser, Z. Zhang, and A. K. Krishna, "Uniform circular broadband beamformer with selective frequency and spatial invariant region," in Proceedings of the 1st International Conference on Signal Processing and Communication System (ICSPCS '07), Gold Coast, Australia, December 2007.

[16] W. Ser, T. T. Zhang, J. Yu, and J. Zhang, "Detection of wheezes using a wearable distributed array of microphones," in Proceedings of the 6th International Workshop on Wearable and Implantable Body Sensor Networks (BSN'09), pp. 296-300, Berkeley, Calif, USA, June 2009.

[17] D. E. N. Davies, "Circular arrays," in Handbook of Antenna Design, Peregrinus, London, UK, 1983.

[18] M. Abramowitz and I. A. Stegum, Handbook of Mathematical Functions, Dover, New York, NY, USA, 1965.

[19] J. Capon, "High-resolution frequency-wavenumber spectrum analysis," Proceedings of the IEEE, vol. 57, no. 8, pp. 1408-1418, 1969.

[20] F. Wang, V. Balakrishnan, P. Y. Zhou, J. J. Chen, R. Yang, and C. Frank, "Optimal array pattern synthesis using semidefinite programming," IEEE Transactions on Signal Processing, vol. 51, no. 5, pp. 1172-1183, 2003.

[21] J. Liu, A. B. Gershman, Z.-Q. Luo, and K. M. Wong, "Adaptive beamforming with sidelobe control: a second-order cone programming approach," IEEE Signal Processing Letters, vol. 10, no. 11, pp. 331-334, 2003.

[22] S. Autrey, "Design of arrays to achieve specified spatial characteristics over broadbands," in Signal Processing, J. W. R. 
Griffiths, Ed., pp. 507-524, Academic Press, New York, NY, USA, 1973.

[23] M. S. Lobo, L. Vandenberghe, S. Boyd, and H. Lebret, "Applications of second-order cone programming," Linear Algebra and Its Applications, vol. 284, no. 1-3, pp. 193-228, 1998.

[24] J. F. Sturm, "Using SeDuMi 1.02, a MATLAB toolbox for optimization over symmetric cones," Optimization Methods and Software, vol. 11, no. 1-4, pp. 625-653, 1999.

[25] S. Yan, Y. Ma, and C. Hou, "Optimal array pattern synthesis for broadband arrays," Journal of the Acoustical Society of America, vol. 122, no. 5, pp. 2686-2696, 2007. 